\title{
Bessaga's conjecture in unstable Köthe spaces and products
}

ZAFER NURLU (Ankara)

and JASSER SARSOUR (Gaza)

Abstract. Let $F$ be a complemented subspace of a nuclear Fréchet space $E$. If $E$ and $F$ both have (absolute) bases $\left(e_{n}\right)$ resp. $\left(f_{n}\right)$, then Bessaga conjectured (see [2] and for a more general form, also [8]) that there exists an isomorphism of $F$ into $E$ mapping $f_{n}$ to $t_{n} e_{\pi\left(k_{n}\right)}$ where $\left(t_{n}\right)$ is a scalar sequence, $\pi$ is a permutation of $\mathbb{N}$, and $\left(k_{n}\right)$ is a subsequence of $\mathbb{N}$. We prove that the conjecture holds if $E$ is unstable, i.e. for some base of decreasing zero-neighborhoods $\left(U_{n}\right)$ consisting of absolutely convex sets one has

$$
\exists s \forall p \exists q \forall r \quad \lim _{n} \frac{d_{n+1}\left(U_{q}, U_{p}\right)}{d_{n}\left(U_{r}, U_{s}\right)}=0
$$

where $d_{n}(U, V)$ denotes the $n$th Kolmogorov diameter.

Introduction and preliminaries. For unexplained terminology the reader is referred to $[6,11,16]$. The cardinality of a set $A$ is denoted by $|A|$.

All spaces considered will be Fréchet spaces, i.e. complete metrizable locally convex topological vector spaces. If $\left(a_{k n}\right)$ is a matrix of nonnegative real numbers satisfying

(i) $\forall n \exists k 0<a_{k n}$,

(ii) $\forall n, k a_{k n} \leq a_{k+1, n}$,

then the sequence space

$$
K\left(a_{k n}\right)=\left\{\left(\xi_{n}\right) \in \mathbb{K}^{\mathbb{N}}:\left\|\left(\xi_{n}\right)\right\|_{k}=\sum_{n}\left|\xi_{n}\right| a_{k_{n}}<\infty \forall k\right\}
$$

is called a Köthe space and the matrix $\left(a_{k n}\right)$ a $K o ̈ t h e ~ m a t r i x . ~ K\left(a_{k n}\right)$ has a continuous norm if and only if $a_{k_{0} n}>0$ for some $k_{0}$ and for all $n$, hence we can assume $a_{k n}>0 \forall n, k . K\left(a_{k n}\right)$ is

(i) nuclear if and only if

$\forall k \exists k^{\prime} \quad\left(a_{k n} / a_{k^{\prime} n}\right) \in \ell_{1} \quad$ (the Grothendieck-Pietsch criterion),

1991 Mathematics Subject Classification: Primary 46A45; Secondary 46A11.

The first author gratefully acknowledges partial support of the group TBAG-CG2 of the Scientific and Technical Research Council of Turkey. 
(ii) called regular if for some matrix $\left(b_{k n}\right)$ generating $K\left(a_{k n}\right)$ one has

$$
\forall n \forall s \leq k \quad b_{k n} / b_{s n} \leq b_{k, n+1} / b_{s, n+1},
$$

(iii) called of type $d_{1}$ if

$$
\exists k \forall j \exists s \quad \sup a_{j n}^{2} /\left(a_{k n} a_{s n}\right)<\infty,
$$

(iv) called of type $d_{2}$ if

$$
\forall k \exists j \forall s \quad \sup a_{k n} a_{s n} /\left(a_{j n}\right)^{2}<\infty,
$$

(v) unstable iff

$$
\exists s \forall p \exists q \forall r \quad \lim _{n} a_{p, n+1} a_{r n} /\left(a_{q, n+1} a_{s n}\right)=0 .
$$

The concept of regularity was introduced by M. M. Dragilev [5] and the conditions $d_{1}$ resp. $d_{2}$ correspond to the conditions $(D N)$ and $(\bar{\Omega})$ of $\mathrm{D}$ Vogt [20] and characterize subspaces with basis of infinite type power series spaces resp. quotients with basis of finite type power series spaces.

Two basic sequences $\left(x_{n}\right)$ resp. $\left(y_{n}\right)$ in Fréchet spaces $X$ resp. $Y$ are called quasi-equivalent (q.e.) if there exists a permutation $\pi$ of $\mathbb{N}$ and scalars $\left(t_{n}\right)$ such that $\sum \xi_{n} x_{n}$ converges in $X$ if and only if $\sum \xi_{n} t_{n} y_{\pi(n)}$ converges in $Y$. If any two unconditional bases in a Fréchet space $E$ are q.e. then $E$ is said to have the quasi-equivalence property, QEP.

Let $E$ be a nuclear Köthe space with basis $\left(e_{n}\right)$ and let $F$ be a complemented subspace of $E$ with basis $\left(f_{r}\right)$. Bessaga showed in [2] that there exists a sequence $\left(k_{n}\right), k_{n} \in \mathbb{N}, \lim k_{n}=\infty$ so that $\left(f_{n}\right)$ is q.e. to $\left(e_{k_{n}}\right)$ and conjectured that:

Conjecture (Bessaga [2]). The sequence $\left(k_{n}\right)$ in the above set-up can be chosen as a subsequence of $\mathbb{N}$ (hence without any repetitions).

Remark 1. Thus in view of the Cantor-Bernstein theorem (see [13]) Bessaga's conjecture is a stronger version of QEP. Even though the validity of QEP is shown for a satisfactorily large class of nuclear Köthe spaces (namely for regular spaces $[3,8]$ and spaces satisfying some weaker regularity conditions), Bessaga's conjecture could be shown to hold only in very special cases; power series spaces $[13,14]$. Dragilev spaces $[1,8]$, regular $d_{1}$ spaces [10], stable regular [3] spaces, and in some very particular cases. It is still not known whether a regular nuclear Köthe space satisfies the conjecture or not. Perhaps it is because of the following obvious relation to the

PEECZYŃsKi PRoBlem. Does every complemented subspace of a nuclear Köthe space have a basis?

Namely, if the answer to the Pełczyński problem is affirmative for a nuclear Köthe space having QEP then it trivially satisfies Bessaga's conjecture.
M. M. Dragilev has shown in [4] that an unstable Fréchet space with basis is regular and using the Grothendieck-Pietsch criterion it is easy to show that it is nuclear. Thus any unstable Fréchet space has QEP. Furthermore, T. Terzioğlu, using a result of J. Krone [12], has shown in [19] that for any unstable Köthe space of type $d_{1}$ or $d_{2}$ the Pełczyński problem has an affirmative answer. So by Remark 1, it follows that any unstable Köthe space of type $d_{1}$ or $d_{2}$ satisfies Bessaga's conjecture. The aim of this paper is to lift the additional assumptions on the type of the basis.

Main results. The proof will be modelled on V. P. Kondakov's method in [10] where he showed that a regular $d_{1}$ nuclear Köthe space satisfies the conjecture. Throughout the section, unless otherwise stated, $E$ will denote an unstable (hence regular, nuclear) Köthe space and $F$ a complemented subspace with basis of $E$. Combining a lemma of V. P. Kondakov [10] and C. Bessaga [2] one can choose a system of fundamental norms so that both conditions below are simultaneously satisfied (see also J. Sarsour [18]).

Lemma 1. Let $\left(e_{n}\right)$ resp. $\left(f_{n}\right)$ be bases of $E$ resp. $F(E, F$ as above). Then there exist regular systems of norms $\left(|\cdot|_{r}\right)$ resp. $\left(\|\cdot\|_{r}\right)$ defining the topologies of $E$ resp. $F$, a continuous projection $Q: E \rightarrow F$, and a nondecreasing sequence $\left(k_{n}\right), k_{n} \in \mathbb{N}$, with $\lim k_{n}=\infty$ such that

$$
\begin{gathered}
|Q e|_{r} \leq 2^{-r}\|Q e\|_{r+1} \leq 2^{-r-2}|e|_{r+1} \quad \forall e \in E \forall r, \\
\frac{\left\|f_{n}\right\|_{r}}{\left\|f_{n}\right\|_{s}}=\frac{\left|e_{k_{n}}\right|_{r}}{\left|e_{k_{n}}\right|_{s}} \quad \forall n, s, r .
\end{gathered}
$$

LEMMA 2. In the set-up of Lemma 1 , if there exist $N$ and a subsequence $\left(i_{n}\right)$ of $\mathbb{N}$ such that $\left(f_{n+N}\right)$ is q.e. to $\left(e_{i_{n}}\right)$ then $\left(f_{n}\right)$ is q.e. to a subsequence of $\left(e_{n}\right)$.

Proof. Since if $\left|\left(e_{n}\right) \backslash\left(e_{i_{n}}\right)\right| \geq N$ then setting up a correspondence is trivially possible, assume the contrary. Then the diametral dimensions of $E$ and $F$ are equal and by a result of Crone-Robinson [3] the result follows.

LEMMA 3. Let $\left(|\cdot|_{r}\right)$ be any regular system of norms defining the topology of an unstable Köthe space $E$ with basis $\left(e_{n}\right)$. Then $\exists s \forall k \geq s \exists q_{k}, M_{k}$ $\forall n>m \geq M_{k}$

$$
\frac{\left|e_{n}\right|_{k+2}}{\left|e_{n}\right| q_{k}}<\frac{\left|e_{m}\right|_{k}}{\left|e_{m}\right|_{q_{k}+2}} \text {. }
$$

Proof. Since $E$ is unstable, from inequality (1) we find $s$. Then for any $k \geq s$ and for $p=k+2$ we find $q=q_{k}$, and take $r=q_{k}+2$. We obtain

$$
\lim _{n} \frac{\left|e_{n+1}\right|_{k+2}}{\left|e_{n+1}\right|_{q_{k}}} \frac{\left|e_{n}\right| q_{k}+2}{\left|e_{n}\right|_{k}}=0 \text {. }
$$


Thus we find $M_{k}$ so that

$$
\frac{\left|e_{n+1}\right|_{k+2}}{\left|e_{n+1}\right|_{q_{k}}}<\frac{\left|e_{n}\right|_{k}}{\left|e_{n}\right|_{q_{k}+2}} \quad \forall n \geq M_{k}
$$

Finally, we replace $n+1$ resp. $n$ with $n$ resp. $m<n$ by regularity.

THEOREM 1. Let $F$ be isomorphic to a complemented subspace with basis $\left(f_{n}\right)$ of a nuclear Köthe space $E$ with basis $\left(e_{n}\right)$. If $F$ is unstable then $\left(f_{n}\right)$ $i s$ q.e. to a subsequence of $\left(e_{n}\right)$.

Proof. By Lemma 1 we find systems of norms $\left(|\cdot|_{r}\right)$ resp. $\left(\|\cdot\|_{r}\right)$ on $E$ resp. $F$ and a sequence $\left(k_{n}\right)$ satisfying in particular the inequality (3). Then by Lemma 3 for some $k$ it follows that for $n \geq M_{k}$ the sequence $\left(k_{n}\right)$ must be without repetitions. Thus by Lemma 2 the conclusion follows.

THEOREM 2. Let $G$ be an unstable Köthe space with a regular representation $G=K\left(a_{k n}\right)$ and let $\left(p_{i}\right), p_{1}=1$, be a subsequence of $\mathbb{N}$. Let $b_{k n}=a_{k i}$ if $p_{i} \leq n<p_{i+1}$. Then $E=K\left(b_{k n}\right)$ satisfies Bessaga's conjecture.

Proof. Since $G=K\left(a_{k n}\right)$ is regular, $E$ is also regular, so it has QEP. Let $F$ be a complemented subspace with basis of $E$. Let $\left(e_{n}\right)$ resp. $\left(f_{n}\right)$ be regular bases in $E$ resp. $F$. So $g_{n}=e_{p_{n}}$ forms a regular basis in $G$. By Lemma 1 we can find systems of norms $\left(|\cdot|_{r}\right)$ resp. $\left(\|\cdot\|_{r}\right)$, defining the topologies of $E$ resp. $F$, which are regular with respect to the mentioned bases and a sequence $\left(k_{n}\right)$ satisfying $(2)$ and (3). By Lemma 3 applied to $G$ with the basis $\left(g_{n}\right)$ we find $s$, fix $r \geq s$ and find $M_{r}$ so that $\forall n>m \geq M_{r}$ the inequality (4) holds. Let

$$
A_{m}=\left\{n \geq p_{M_{r}}: \frac{\left\|f_{m}\right\|_{r}}{\left\|f_{m}\right\|_{t+4}} \leq \frac{\left|e_{n}\right|_{r}}{\left|e_{n}\right|_{t+2}} \leq \frac{\left\|f_{m}\right\|_{r}}{\left\|f_{m}\right\|_{t}} \quad \forall t \geq r\right\} .
$$

Then by $(3)$, it follows that $A_{m} \neq \emptyset$. Let $m_{1}, \ldots, m_{k}$ be given distinct indices. Following Kondakov's method [10] we are going to show that

$$
\left|\bigcup_{i=1}^{k} A_{m_{i}}\right| \geq k
$$

and then apply the combinatorial Hall-Koenig theorem [7] to choose distinct; representatives from each $A_{m}$. By regularity each $A_{m}$ consists of an interval of natural numbers and if $\bigcup_{i=1}^{k} A_{m_{i}}$ consists of disjoint intervals then the result easily follows once we show it for each interval. Thus without loss of generality we suppose

$$
B=\bigcup_{i=1}^{k} A_{m_{i}}=\left\{n: \frac{\left|e_{N_{k}}\right|_{r}}{\left|e_{N_{k}}\right|_{s+4}} \leq \frac{\left|e_{n}\right|_{r}}{\left|e_{n}\right|_{s+2}} \leq \frac{\left|e_{N_{1}}\right|_{r}}{\left|e_{N_{1}}\right|_{s}} \forall s \geq r\right\} .
$$

where $N_{k}=\max \left\{n_{m_{i}}: 1 \leq i \leq k\right\}$ and $N_{1}=\min \left\{n_{m_{i}}: 1 \leq i \leq k\right\}$, that is, we suppose that $B$ is the interval $\left[N_{1}, N_{k}\right]$. If $p_{\alpha} \leq N_{1}<p_{\alpha+1}$ and $p_{\beta} \leq N_{k}<p_{\beta+1}$ then $\alpha, \beta \geq M_{r}$ and

$$
B=\left\{n: \frac{\left|g_{\beta}\right|_{r}}{\left|g_{\beta}\right|_{s+4}} \leq \frac{\left|e_{n}\right|_{r}}{\left|e_{n}\right|_{s+2}} \leq \frac{\left|g_{\alpha}\right|_{r}}{\left|g_{\alpha}\right|_{s}} \quad \forall s \geq r\right\} .
$$

We let

$$
H=\left\{m: \frac{\left|g_{\beta}\right|_{t}}{\left|g_{\beta}\right|_{s}} \leq \frac{\left\|f_{m}\right\|_{t}}{\left\|f_{m}\right\|_{s}} \leq \frac{\left|g_{\alpha}\right|_{t}}{\left|g_{\alpha}\right|_{s}} \quad \forall s \geq t\right\} .
$$

Since $m_{1}, \ldots, m_{k} \in H,|H| \geq k$. We claim that $|B| \geq|H|$. If not, then we find $0 \neq h \in F$ such that

$$
h=\sum_{n \notin B} e_{n}^{\prime}(h) e_{n}=\sum_{m \in H} f_{m}^{\prime}(h) f_{m}
$$

where $e_{n}^{\prime}, f_{m}^{\prime}$ denote the associated coordinate functionals of $\left(e_{n}\right)$ resp. $\left(f_{m}\right)$. Let

$$
\begin{aligned}
& B_{1}=\left\{n: e_{n}^{\prime}(h) \neq 0 \text { and } \exists \xi_{n} \text { with } \frac{\left|e_{n}\right|_{r}}{\left|e_{n}\right| \xi_{n}+2}<\frac{\left|g_{\beta}\right|_{r}}{\left|g_{\beta}\right|_{\xi_{n}+4}}\right\}, \\
& B_{2}=\left\{n: n \notin B_{1}, e_{n}^{\prime}(h) \neq 0 \text { and } \exists \eta_{n} \text { with } \frac{\left|e_{n}\right|_{r}}{\left|e_{n}\right|_{\eta_{n}+2}}>\frac{\left|g_{\alpha}\right|_{r}}{\left|g_{\alpha}\right|_{\eta_{n}}}\right\} .
\end{aligned}
$$

Thus $B_{1}$ resp. $B_{2}$ denotes the set of indices where the first resp. second inequality in $B$ does not hold. Let $n \in B_{1}$. Then for some $i_{n}$ we have $p_{i_{n}} \leq n<p_{i_{n}+1}$ and $\left|e_{n}\right|_{r}=\left|g_{i_{n}}\right|_{r}$. We have

$$
\frac{\left|g_{i_{n}}\right|_{r}}{\left|g_{i_{n}}\right|_{\xi_{n}+2}}=\frac{\left|e_{n}\right|_{r}}{\left|e_{n}\right|_{\xi_{n}+2}}<\frac{\left|g_{\beta}\right|_{r}}{\left|g_{\beta}\right|_{\xi_{n}+4}}
$$

and thus by regularity $i_{n}>\beta$ and by inequality (4)

$$
\frac{\left|e_{n}\right|_{r+2}}{\left|e_{n}\right|_{q_{r}}}=\frac{\left|g_{i_{n}}\right|_{r+2}}{\left|g_{i_{n}}\right|_{q_{r}}}<\frac{\left|g_{\beta}\right|_{r}}{\left|g_{\beta}\right|_{q_{r}+2}} \quad \text { for } n \in B_{1} .
$$

Let now $n \in B_{2}$. Then for some $j_{n}$ we have $p_{j_{n}} \leq n<p_{j_{n}+1}$ so $\left|e_{n}\right|_{r}=\left|g_{j_{n}}\right|_{r}$. We have

$$
\frac{\left|g_{j_{n}}\right|_{r}}{\left|g_{j_{n}}\right| \eta_{n}+2}=\frac{\left|e_{n}\right|_{r}}{\left|e_{n}\right|_{\eta_{n}+2}}>\frac{\left|g_{\alpha}\right|_{r}}{\left|g_{\alpha}\right|_{\eta_{n}}} \geq \frac{\left|g_{\alpha}\right|_{r}}{\left|g_{\alpha}\right|_{\eta_{n}+2}}
$$

and thus by regularity $j_{n}<\alpha$ and by (4)

$$
\frac{\left|g_{\alpha}\right|_{r+2}}{\left|g_{\alpha}\right|_{q_{r}}}<\frac{\left|g_{j_{n}}\right|_{r}}{\left|g_{j_{n}}\right| q_{r}+2}=\frac{\left|e_{n}\right|_{r}}{\left|e_{n}\right|_{q_{r}+2}} \quad \text { for } n \in B_{2} \text {. }
$$

$$
h_{i}=\sum_{n \in B_{i}} e_{n}^{\prime}(h) \sum_{m \in H} f_{m}^{\prime}\left(Q e_{n}\right) f_{m}, \quad i=1,2 .
$$

Then $h=h_{1}+h_{2}$. We have 


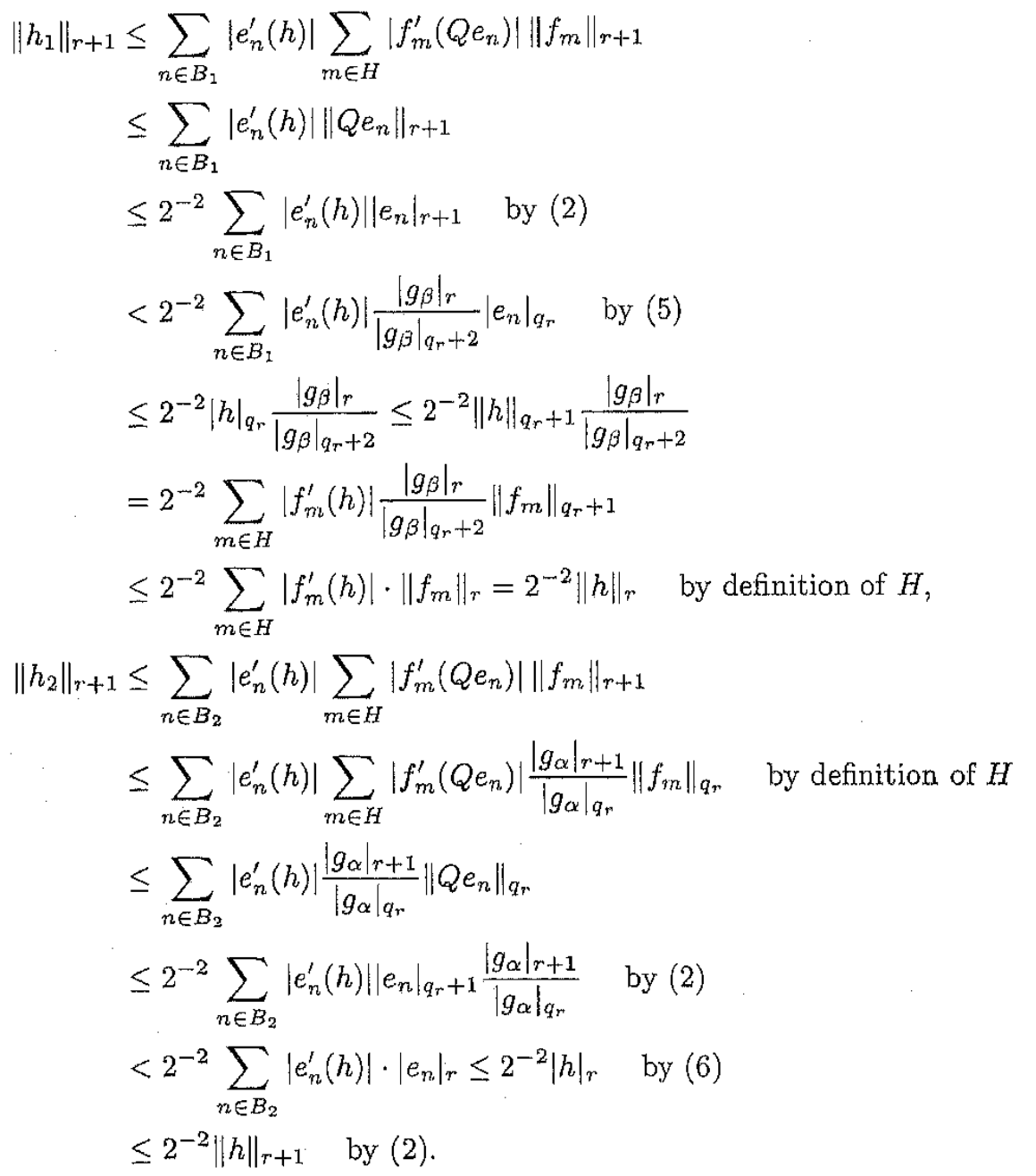

Thus we get the contradiction that

$$
0 \neq\|h\|_{r+1} \leq\left\|h_{1}\right\|_{r+1}+\left\|h_{2}\right\|_{r+1} \leq \frac{1}{2}\|h\|_{r+1} .
$$

Finally, using the Hall-Koenig theorem we choose distinct indices $\left(n_{m}\right)$ with $n_{m} \in A_{m}$. Then with $t_{m}=\left\|f_{m}\right\|_{r} /\left|e_{n_{m}}\right|_{r}$ we get

$$
\left\|f_{m}\right\|_{t} \leq t_{m}\left|e_{n_{m}}\right|_{t+2} \leq\left\|f_{m}\right\|_{t+4} \quad \forall t \geq r, m \geq M_{r} .
$$

The conclusion follows by Lemma 2 .

Corollary 1. Every unstable Köthe space satisfies Bessaga's conjecture.
COROLLARY 2. Every complemented subspace with basis of an unstable Köthe space is unstable.

In the remainder of the paper we investigate the direct sum of two "unlike" spaces with regard to Bessaga's conjecture. Write $(E, F) \in \mathbf{K}$ if all linear continuous maps $T: E \rightarrow F$ are compact.

Lemma 4. Let $E=K\left(a_{k n}\right), F=K\left(b_{k n}\right)$ be nuclear, have continuous norm, and assume $(E, F) \in \mathbf{K}$. For a subsequence $\left(n_{p}\right)$ of $\mathbb{N}$ define $m_{n}=p$ if $n_{p-1} \leq n<n_{p}$. Then assuming all considered spaces are nuclear, one has:

(1) $\left(K\left(a_{k m_{n}}\right), K\left(b_{k n}\right)\right) \in \mathbf{K}$,

(2) $\left(K\left(a_{k n}\right), K\left(b_{k m_{n}}\right)\right) \in \mathbf{K}$

Proof. See [15].

THEOREM 3. Let $E_{1}$ and $E_{2}$ be nuclear Köthe spaces satisfying Bessaga's conjecture and $\left(E_{1}, E_{2}\right) \in \mathbf{K}$. Then if $E=E_{1} \oplus E_{2}$ is either isomorphic to a finite-codimensional subspace of itself or is not isomorphic to any proper subspace of itself then $E$ satisfies Bessaga's conjecture.

Pr o of. Since in particular $E_{1}$ and $E_{2}$ have QEP, so does $E$ by a result of V. P. Kondakov [9]. Let $\left(e_{n}^{1}\right)$ resp. $\left(e_{n}^{2}\right)$ be bases in $E_{1}$ resp. $E_{2}$. Thus $\left(e_{n}\right)=$ $\left(e_{n}^{1}\right) \cup\left(e_{n}^{2}\right)$ is a basis in $E$. Let $F$ be a complemented subspace with basis $\left(f_{n}\right)$ of $E$. By C. Bessaga's crucial lemma [2] there exists a nondecreasing sequence $\left(m_{n}\right), m_{n} \in \mathbb{N}$, with $\left(f_{n}\right)$ q.e. to $\left(e_{m_{n}}\right)$. Let $\left(f_{i n}\right)_{n}=\left\{f_{j}: e_{m_{j}} \in\left(e_{n}^{i}\right)_{n}\right\}$ and $F_{i}=\overline{\operatorname{span}\left(f_{i n}\right)}$ for $i=1,2$. Then $F_{1}$ and $F_{2}$ are complemented subspaces of $E$ and by Lemma 4 also $\left(F_{1}, E_{2}\right) \in \mathbf{K}$ and $\left(E_{1}, F_{2}\right) \in \mathbf{K}$. Since $F_{1}$ is a complemented subspace of $E_{1} \oplus E_{2}$ and $\left(E_{1}, E_{2}\right) \in \mathbf{K}$, by a result of J. Prada [17] $F_{1}$ is isomorphic to $D_{1} \oplus D_{2}$ where $D_{i}$ is a complemented subspace of $E_{i,}, i=1,2$. It follows that $D_{2}$ is finite-dimensional. Let $\left(d_{n}\right)$ be a basis of $D_{1}$ which is q.e. to $\left(f_{1, n+M_{1}}\right)_{n}$ for some $M_{1}$. Analogously we find $M_{2}$ and a basis $\left(c_{n}\right)$ in a complemented subspace $C_{2}$ of $E_{2}$ with $\left(c_{n}\right)$ q.e. to $\left(f_{2, n+M_{2}}\right)_{n}$. Since $E_{1}$ and $E_{2}$ satisfy Bessaga's conjecture we deduce that $\left(f_{1, n+M_{1}}\right) \cup\left(f_{2, n+M_{2}}\right)$ is q.e. to $\left(d_{n}\right) \cup\left(c_{n}\right)$ which is q.e. to a subsequence of $\left(e_{n}\right)$. The conclusion follows easily from the assumptions on $E$.

Remark 2. The assumptions on $E=E_{1} \oplus E_{2}$ in Theorem 3 can be replaced by the regularity of $E$ or $F$ (see Crone-Robinson [3]).

Remark 3 . The authors are informed that using different techniques V. P. Kondakov has recently obtained the yet unpublished result that the Pełczyński problem has an affirmative answer for unstable spaces with basis. Since such spaces are known to have QEP, our Theorem 2 would become a consequence of Kondakov's result. 


\section{References}

[1] H. Ahonen, On nuclear spaces defined by Dragilev functions, Ann. Acad. Sci. Fenn. Ser. Al Math. Dissertationes 38 (1981), 1-57.

2] C. Bessaga, Some remarks on Dragilev's theorem, Studia Math. 31 (1968), 307318.

[3] L. Crone and W. B. Robinson, Every nuclear Frěchet space with a regular basis has the quasi-equivalence property, ibid. 52 (1975), 203-207.

(4] M. M. Dragilev, On special dimensions defined on some classes of Köthe spaces, Math. USSR-Sb. 9 (2) (1968);213-228.

[5] - On regular bases in nuclear spaces, Amer. Math. Soc. Transl. (2) 93 (1970) 61-82.

6] E. Dubinsky, The Structure of Nuclear Fréchet Spaces, Lecture Notes in Math. 720, Springer, Berlin 1979.

7] M. Hall, Jr., Combinatorial Theory, Blaisdell-Waltham, 1967.

[8] V. P. Kondakov, On a certain generalization of power series spaces, in: Current Problems in Mathematical Analysis, Rostov State Univ, 1978, 92-99 (in Russian).

- Properties of bases of some Köthe spaces and their subspaces, in: Functional Analysis and its Applications 14, Rostov State Univ., 1980, 58-59 (in Russian).

[10] -, Unconditional bases in certain. Köthe spaces, Sibirsk. Mat. Zh. 25 (3) (1984), 109-119 (in Russian).

[11] G. Köthe, Topologische lineare Räume I, Springer, Berlin 1960.

[12] J. Krone, Bases in the range of operators between Köthe spaces, Doğa Tr. J. Math. 10 (1986), 162-166 (special issue).

[13] B. S. Mityagin, Approximative dimension and bases in nuclear spaces, Uspekhi Mat. Nauk 16 (4) (1961), 73-132 (in Russian).

[14] —, Equivalence of bases in Hilbert scales, Studia Math. 37 (1971), 111-137 (in Russian).

[15] Z. Nurlu, On pairs of Köthe spaces between which all operators are compact, Math. Nachr. 122 (1985), 272-287.

16] A. Pietsch, Nuclear Locally Convex Spaces, Springer, Berlin 1972.

[17] : J. Prada, On idempotent operators on Fréchet spaces, Arch. Math. (Basel) 43 (1984), 179-182.

[18] J. Sarsour, Bessaga's conjecture and quasi-equivalence property in unstable Köthe spaces, Ph.D. Thesis, METU, Ankara 1991.

[19] T. Terzioğlu, Unstable Köthe spaces and the functor Ext, Doğa Tr. J. Math. 10 (1986), 227-231 (special issue).

20] D. Vogt, Eine Charakterisierung der Potenzreihenräume von endlichem Typ und ihre Folgerungen, Manuscripta Math. 37 (1982), 269-301.
DEPARTMENT OF MATHEMATICS MIDDLE EAST TECHNICAL UNIVERSTTY 06531 ANKARA, TURKEY
DEPARTMENT OF MATHFMATTCS

$$
\text { P.O. BOX } 108
$$

GAZA, GAZA THE ISLAMIC UNIVERSTTY OF GAZA

\section{Semigroups with nonquasianalytic growth}

by

VŨ QUÔC PHÓNG (Hanoi)

Abstract. We study asymptotic behavior of $C_{0}$-semigroups $T(t), t \geq 0$, such that $\|T(t)\| \leq \alpha(t)$, where $\alpha(t)$ is a nonquasianalytic weight function. In particular, we show that if $\sigma(A) \cap i \mathbb{R}$ is countable and $P \sigma\left(A^{*}\right) \cap i \mathbb{R}$ is empty, then $\lim _{t \rightarrow \infty} \frac{1}{\alpha(t)}\|T(t) x\|=0$, $\forall x \in X$. If, noreover, $f$ is a function in $L_{\alpha}^{1}\left(\mathbb{R}_{+}\right)$which is of spectral synthesis in a corresponding algebra $L_{\cap_{1}}^{1}(\mathbb{R})$ with respect to $(i \sigma(A)) \cap \mathbb{R}$, then $\lim _{t \rightarrow \infty} \frac{1}{\alpha(t)}\|T(t) \widehat{f}(T)\|=$ 0 , where $\widehat{f}(T)=\int_{0}^{\infty} f(t) T(t) d t$.

Analogous results are obtained also for iterates of a single operator. The results are extensioms of earlier results of Katznelson-Tzafriri, Lyubich-Vü Quôc Phóng, Arendt Batty ..., concerning contraction semigroups. The proofs are based on the operator form of the Tauberian Theorem for Beurling algebras with nonquasianalytic weight.

0. The classical Wiener Approximation Theorem is equivalent to the General Tauberian Theorem (see e.g. [5, 10]). In the papers [11], [12], we have shown that from this theorem one can obtain some new results (or new proofs of old results) on asymptotic behavior of iterates of power-bounded operators, and of trajectories of bounded $C_{0}$-semigroups (cf. $[1,2,6,8]$ ). On the other hand, Beurling has introduced a more general class of Banach algebras $L_{\alpha}^{1}(\mathbb{R})$ with a weight $\alpha$, and proved that the Tauberian Theorem remains valid if $\alpha$ satisfies a certain condition of nonquasianalyticity (see e.g. [10]). This theorem has then been used in [7] for spectral analysis of groups of operators which are dominated by nonquasianalytic weight functions.

In this paper, we extend the results of $[1,2,6,8,11]$ to a more general case of semigroups $\mathcal{T}=\{T(t): t \geq 0\}$ of bounded linear operators such that $\|T(t)\|$ is dominated by a weight function with nonquasianalytic growth. We use an operator-theoretical form of the Tauberian Theorem (Lemmas 5 and 6), and a modification of a construction (limit semigroups) in $[11]$.

1991 Mathematics Subject Classification: Primary 47D05; Secondary 47 A10. 